\title{
Effect of Heterogeneity of Hydraulic Conductivity on Streaming Potential
}

\author{
Yusuke OZAKI ${ }^{1}$, Hitoshi MIKADA ${ }^{1}$ Tadanori GOTO $^{1}$ Junichi TAKEKAWA $^{1}$ \\ Maki TSUJIMURA ${ }^{2}$ and Fatma HACHANI ${ }^{2}$ \\ ${ }^{1}$ Dept. of Civil and Earth Res. Eng., Kyoto University \\ ${ }^{2}$ Dept of Life and Environmental Sciences, University of Tsukuba
}

\begin{abstract}
Self-potential(SP) is electrical potential mainly generated by thermoelectric, chemical and streaming potentials in the subsurface. The flow of groundwater is often recognized as a bigger source of SP. Using this feature, there are many attempts to localize and quantify flows of liquid in the soil, including groundwater. In case that underground structure is homogeneous, electrical current density according to ground-water flow becomes uniform. Therefore SP on the surface increases monotonically from upstream to downstream of groundwater flow. As a basic interpretation of SP, the direction of increase in SP corresponds with the direction of groundwater flow. However, the anomalous fluctuations of SP start to appear for subsurface inhomogeneous groundwater flow due to the non-uniform conveyance of changes. Extra charge occurs on the boundary of these parameters. As a result, local minimum or maximum in the profile of SP generate just above the boundaries. Our simulation shows that the anomalies of permeability and coupling-coefficient in the subsurface are predominant parameters to effectively estimate the distribution of surface SP in the existence of inhomogeneous underground flow. The effect of coupling coefficient on the SP is often simulated on the underground flow scale. Some anomalies of SP are explained by the inhomogeneous of coupling coefficient. Simple model simulation, for example the well-pump model shows the effect of hydraulic conductivity on the SP. However, SP anomalies that are generated by inhomogeneity of hydraulic conductivity are not simulated on the underground flow scale. We compare the difference of these anomalies and study the different feature of SP anomaly that is generated by hydraulic conductivity from it by coupling coefficient.
\end{abstract}

\section{INTRODUCTION}

SP is electrical potential that is generated on the surface and subsurface. Self-potential method is method that measure distribution of this potential. There are some sources of SP and groundwater flow is one of the biggest factors. Many SP measurements have been conducted because it is easy to measure SP and the cost of acquisition of the data is low.

SP distribution around the pumped well would be generated by groundwater flow ${ }^{1)}$. There are many measurements to acquire the data that shows the existence of groundwater. However, only the SP is sensitive to ground flow. The information of groundwater flow and structure of hydraulic conductivity could be predictable from $\mathrm{SP}^{2)}$.

Uniform ground structure is often supposed on the interpretation of SP. In this case, the fluctuations of SP increase monotonously from intrusion to spring. However, complex patterns of SP distribution have been measured. These patterns would be due to complex groundwater flow due to inhomogeneous underground. However, the effects of inhomogeneity on the SP have not been considered sufficiently. To acquire the information of flow correctly, it is necessary to estimate this effect on SP.

In this study, we simulate the groundwater flow and the SP due to this flow. We estimate the effect of underground structure on SP distribution. Hydraulic conductivity, coupling co-efficient and electrical conductivity are parameters in this study. We searched which parameter rules the SP distribution.

\section{THEORY}

As shown in Fig.1, anion and cation exist separately on the surface of particles with water. Cations are usually included outer the shear plane where groundwater can flow. Cation outer the shear plane is conveyed by ground water flow and this 
generates current density. In case that gradient of geothermal heat is loose and concentration of ion are enough weak, only the groundwater pressure convey ion. Electrical current density that is generated by groundwater flow is in proportion to the gradient of pressure head and dominated by the ohm's law. Groundwater flow depends on the gradient of pressure head and electrical potential. These can be described by equation (1) and (2). These equations show that electrical current density $\mathrm{I}$ and the flow of water $\mathrm{J}$ are connected with electrical potential $\varphi$ and hydraulic head $\xi$. L are co-efficient of transportation and they are electrical conductivity, coupling co-efficient and permeability from equation(1).

$$
\begin{aligned}
& I=-L_{e e} \nabla \varphi-L_{e v} \nabla \xi \\
& J=-L_{e v} \nabla \varphi-L_{v v} \nabla \xi
\end{aligned}
$$

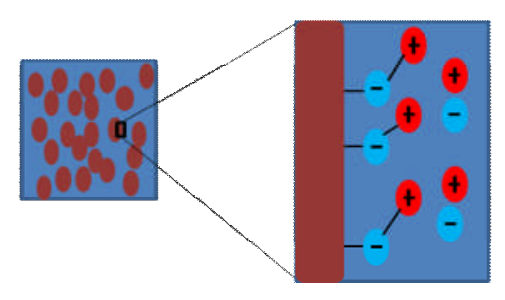

Fig1. The image of surface of particle.

The effect of electrical field on groundwater can be negligible safely, on condition that permeability is larger than $10^{-15}$.) Groundwater flow is dominated by the Darcy's law.

The law of conservation of electrical charge must be satisfied. The equation (3) that connects hydraulic head and electrical potential is derived from equation(1) and law of conservation.

$$
\nabla \sigma \nabla \varphi=-\rho g\left(\nabla L \nabla h+L \nabla^{2} h\right)
$$

where $\sigma$ is electrical conductivity, $\varphi$ is electrical potential, $\rho$ is water density, $L$ is coupling-coefficient and $\mathrm{h}$ is hydraulic head.

\section{METHOD}

In this study, we calculated the SP by three steps.

(i) Calculation of hydraulic head from distribution of permeability and boundary condition.

(ii) Calculation of distribution of electrical charge from hydraulic head and coupling co-efficient (iii) Calculation of the distribution of electrical potential from electrical charge and electrical conductivity.

We developed $2 \mathrm{~d}$ finite differential method code. Heterogeneity of underground structure is located in the groundwater flow as shown in Fig.2 to estimate the effect of this on SP.

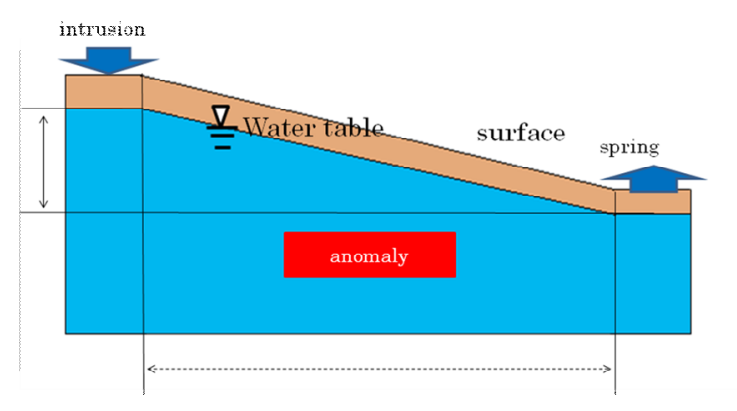

Fig2. The simulation model

\section{RESULT}

\section{(1)Effect of coupling co efficient on SP}

Fig.3 is the result of SP on the condition that coupling co-efficient is $2.5 \times 10^{-9}, 5.0 \times 10^{-9}, 2.0 \times 10^{-8}$ and $4.0 \times 10^{-8} \mathrm{~A} / \mathrm{Pa} \mathrm{m}$. The width of model is $2 \mathrm{~km}$ and the difference of hydraulic head is $5 \mathrm{~m}$. The anomaly structure has $1 \mathrm{~km}$ width and is located in the center of the model. Permeability is $1.0 \times 10^{-6} \mathrm{~m} / \mathrm{s}$ and electrical conductivity is $0.01 \mathrm{~S} / \mathrm{m}$ uniformly. Coupling co-efficient outside anomaly is $1.0 \times 10^{-8}$ $\mathrm{A} / \mathrm{Pa} \mathrm{m}$. Groundwater flow from left to right.

The graph (o) in the Fig. 3 is the SP in case that underground structure is uniform. SP distribution increases monotony with the same direction of groundwater flow.

On condition that there is anomaly, anomaly of SP distribution appears just above the boundary of structural anomaly. The peak of anomaly is in proportion to the difference of material property. The peak is plus on the boundary where inhomogeneity has larger coupling coefficient than around and minus where inhomogeneity has smaller.

\section{(2)Effect of hydraulic conductivity on SP}

Fig.4 is the result of SP on the condition that the permeability of anomaly is larger than around. Fig.5 shows the distribution of electrical charge in this case. Permeability of anomaly is $4.0 \times 10^{-6} \mathrm{~m} / \mathrm{s}$. The width of model is $800 \mathrm{~m}$. The structural anomaly has $800 \mathrm{~m}$ width and is located in the center of the model. Coupling co-efficient is $1.0 \times 10^{-8} \mathrm{~A} / \mathrm{Pa} \mathrm{m}$ and electrical conductivity is $0.01 \mathrm{~S} / \mathrm{m}$ uniformly. Permeability outside anomaly is $1.0 \times 10^{-6} \mathrm{~m} / \mathrm{s}$. Groundwater flow from left to right. The graph includes the result that the difference of hydraulic 
head is $10 \mathrm{~m}, 25$ and $40 \mathrm{~m}$.

Electrical charge occurs on the boundary of the structure and the anomaly of SP distribution appears just above the boundary of structure. The peak of anomaly of SP is proportion to the difference of hydraulic head.

Fig.6 is the result of SP on the condition that the permeability of the anomaly is smaller than around. Fig. 7 shows the distribution of electrical charge in this case. Permeability of the anomaly is $2.5 \times 10^{-7}$ $\mathrm{m} / \mathrm{s}$. The model and the other parameters are same as Fig.4.

In this case, flow of groundwater changes and much electrical charge occur on the upper and the lower boundary than the side boundary. As a result, the fluctuation of SP is small.

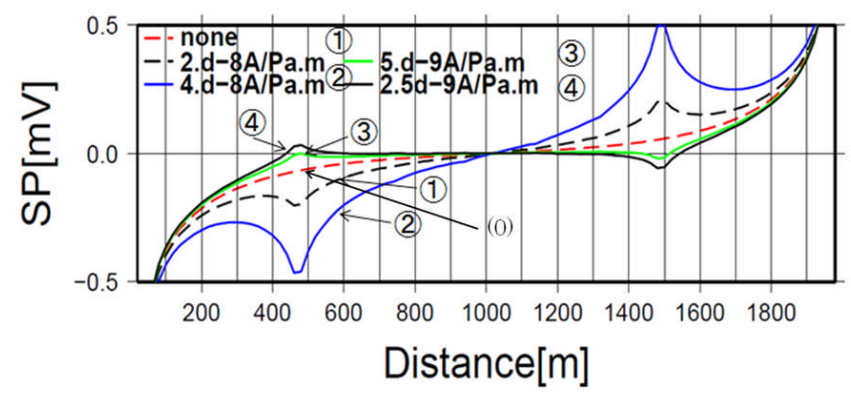

Fig.3 The result of SP on the condition that coupling co-efficient of anomaly is different.

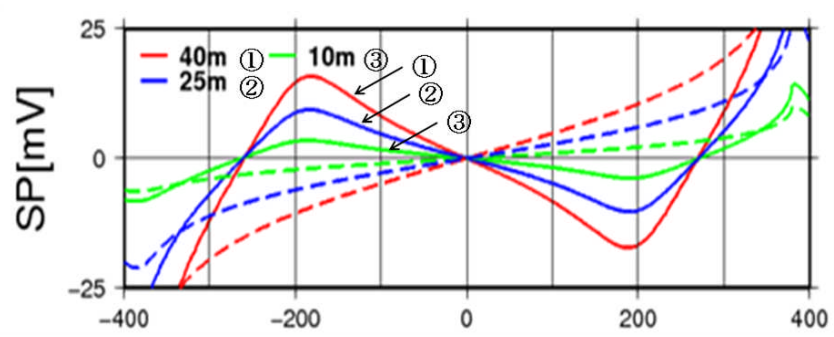

Fig.4 The result of SP on the condition that permeability of anomaly is larger than around.

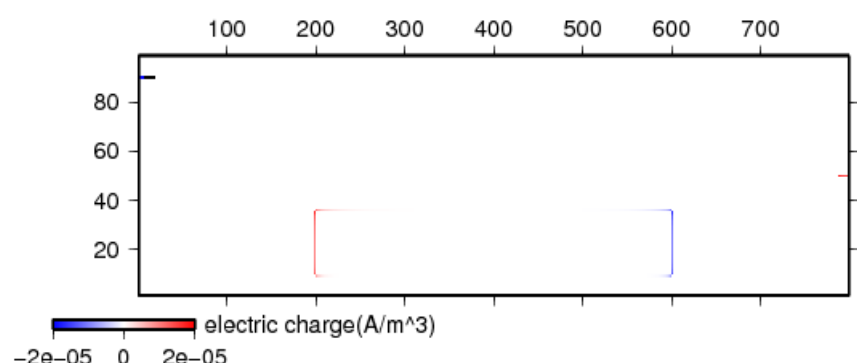

Fig.5 The distribution of electrical charge on the condition that permeability of anomaly is larger than around

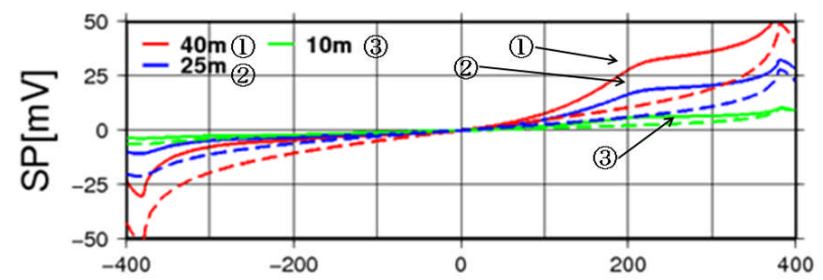

Fig.6 The result of SP on the condition that permeability of anomaly is smaller than around.

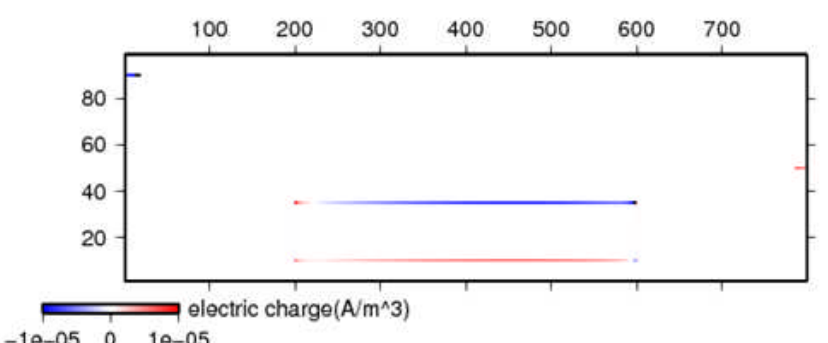

Fig. 7 The distribution of electrical charge on the condition that permeability of anomaly is smaller than around

\section{CONCLUSION}

In this study, we developed simulation program and estimated the effect of structural inhomogeneity in the groundwater flow on the SP.

Simulation results show that coupling co-efficient and permeability are the most effective parameters on the SP. In case that groundwater flow is horizontal, anomaly of the SP appears just above the boundary of structure. Qualitative estimation that there exists structural boundary just under the peak of the SP distribution could be done from that.

Anomaly of permeability changes groundwater flow. The SP distribution could be complex due to this. To estimate parameters quantitatively from SP distribution, detailed simulation would be important.

\section{REFERENCES}

1) Titov K , A. Revil , P.Konosavsky , S.Straface and S. Troisi(2005): Numerical modeling of self-potential signals associated with a pumping test experiment, Geophys. J. Int 162, 641-650

2) Mathieu Darnet and Guy Marquis(2004): Modelling of streaming potential(SP) signals induced by water movement in the vadose zone , Journal of Hydrology 285, 114-124.

3) Tsuneo Ishido and John W.Pritchett(1999): Numerical simulation of electrokinetic potential associated with subsurface fluid flow, Jornal of Geophysical Research,104,247-259. 